\title{
Therapeutic Outcomes of Endoscopic Resection of Early Gastric Cancer with Undifferentiated-Type Histology: A Korean ESD Registry Database Analysis
}

\author{
Chang Seok Bang ${ }^{1}$, Jae Myung Park², Gwang Ho Baik ${ }^{1 *}$, Jong Jae Park ${ }^{3^{*}}$, Moon Kyung Joo ${ }^{3}$, Jae Young Jang ${ }^{4}$, Seong Woo Jeon ${ }^{5}$, \\ Suck Chei Choi ${ }^{6}$, Jae Kyu Sung ${ }^{7}$ and Kwang Bum $\mathrm{Cho}^{8}$ \\ ${ }^{1}$ Department of Internal Medicine, Hallym University College of Medicine, Chuncheon, ${ }^{2}$ Department of Internal Medicine, College of Medicine, \\ The Catholic University of Korea, Seoul, ${ }^{3}$ Department of Internal Medicine, Korea University College of Medicine, Seoul, ${ }^{4}$ Department of \\ Internal Medicine, Kyung Hee University College of Medicine, Seoul, ${ }^{5}$ Department of Internal Medicine, Kyungpook National University \\ School of Medicine, Daegu, ${ }^{6}$ Department of Internal Medicine and Digestive Disease Research Institute, Wonkwang University School of \\ Medicine, Iksan, ${ }^{7}$ Department of Internal Medicine, Chungnam National University School of Medicine, Daejeon, ${ }^{8}$ Department of Internal \\ Medicine, Keimyung University School of Medicine, Daegu, Korea
}

Background/Aims: To assess the therapeutic outcomes of endoscopic resection (ER) of early gastric cancer (EGC) with undifferentiated-type histology.

Methods: Cases of ER of EGC with undifferentiated-type histology in the Korean endoscopic submucosal dissection (ESD) registry database were identified and reviewed. The immediate outcomes, including en bloc resection, complete resection, and curative resection rates, and long-term outcomes, including recurrence and survival rates, were extracted and analyzed.

Results: From 2006 to 2015, 275 EGCs with undifferentiated-type histology from 275 patients were identified. The immediate outcomes were as follows: en bloc resection rate: $92.4 \%$; complete resection rate: $80 \%$; and curative resection rate: $36.4 \%$. Compared to patients with lesions that were beyond the expanded indication, those with expanded indication lesions showed better therapeutic outcomes. There was no difference in immediate outcomes between patients with poorly differentiated adenocarcinoma (PDC) and signet ring cell carcinoma (SRC). However, compared to ER of SRC, ER of PDC had a stronger association with submucosal invasion (41.9\% vs. $23.6 \%$, $p=0.003$ ). With regard to long-term outcomes, there was no difference between lesions with curative and non-curative resections in the recurrence and mortality rates. These rates also did not differ between PDC and SRC (median follow up: 3.96 years).

Conclusions: ER confined to expanded indication lesions can be considered for treatment of EGC with undifferentiated-type histology.

Clin Endosc 2017;50:569-577

Key Words: Endoscopic mucosal resection; Stomach neoplasms; Undifferentiated

\section{INTRODUCTION}

Endoscopic resection (ER) of early gastric cancer (EGC) has

Received: January 8, 2017 Revised: February 23, 2017 Accepted: March 6, 2017

Correspondence: Gwang Ho Baik

Department of Internal Medicine, Hallym University College of Medicine, Chuncheon Sacred Heart Hospital, 77 Sakju-ro, Chuncheon 24253, Korea Tel: +82-33-240-5821, Fax: +82-33-241-8064, E-mail: baikgh@hallym.or.kr

*These authors equally contributed to this work

(cc) This is an Open Access article distributed under the terms of the Creative Commons Attribution Non-Commercial License (http://creativecommons.org/ licenses/by-nc/3.0) which permits unrestricted non-commercial use, distribution, and reproduction in any medium, provided the original work is properly cited. been established as a primary treatment option for a specific subset of patients who have negligible risk of lymph node metastasis. ${ }^{1,2}$ With technical advancements in ER, including endoscopic mucosal resection (EMR) or endoscopic submucosal dissection (ESD), the indications for ER of EGC have been expanded. ${ }^{1-3}$ However, EGCs with undifferentiated-type histology, including poorly differentiated adenocarcinoma (PDC) or signet ring cell carcinoma (SRC), have distinctive growth patterns, and ER for these lesions is still considered an investigational treatment. $^{4-6}$

The therapeutic outcomes of ER of EGC with undifferentiated-type histology have previously been presented using a 
meta-analysis of small studies. ${ }^{7}$ Although these data suggested the technical feasibility of ER of these lesions, the follow-up durations of the included studies were relatively short, and most studies did not present therapeutic outcomes divided by PDC and SRC. Moreover, inconsistent implementation of pre-ER indications for these lesions was the reason for heterogeneity among the studies, making interpretation of pooled estimates unclear.

Therefore, this study evaluated the therapeutic outcomes of ER of EGC with undifferentiated-type histology, using the multicenter ESD registry database, in order to determine the suitability of current indications for ER.

\section{MATERIALS AND METHODS}

\section{Study population}

The Korean ESD registry database is an online registry created in 2015. It is an ongoing project collecting ESD or EMR data from multiple centers representing different geographical areas in Korea (https://esd.gie.or.kr) and is under quality control of the Korean Society of Gastrointestinal Endoscopy. This registry contains clinical information, endoscopic findings, therapeutic outcomes, and follow-up data, including recurrence and survival related to ER of gastric neoplasms. Cases with ER of EGCs with undifferentiated-type histology were identified and reviewed. Clinical, endoscopic, and histologic findings before and after ER were evaluated retrospectively from the original database.

\section{ER and surveillance}

ESD or EMR procedures were performed using standard techniques (marking, submucosal injection, incision, submucosal dissection/injection, and snaring), although different types of instruments, including endoscopes, electrosurgical units, sedative drugs, and endoscopic knives were used. ER of small (less than $2 \mathrm{~cm}$ ) non-ulcerative intramucosal gastric cancer with undifferentiated-type histology was defined as expanded indication or criteria, according to Korean guidelines. ${ }^{5}$ ER of EGCs with undifferentiated-type histology that did not meet this standard was defined as beyond expanded indication or criteria. Pre-ER indication refers to the judgment of the endoscopist as to whether the lesion is candidate for ER or not, according to the guidelines. ${ }^{5}$ Post-ER criteria is the result of histologic evaluation as to whether the resected lesion is included in the expanded or beyond expanded criteria. ${ }^{5}$

Follow-up endoscopic surveillance was performed every 3 to 6 months for 1-2 years and every 6 months for the next $3-5$ years, according to the national comprehensive cancer network (NCCN) guidelines. ${ }^{8}$ Abdominal and pelvic computed tomography using a contrast, and chest X-ray were performed every 6 months for 5 years.

\section{Therapeutic outcomes}

Immediate therapeutic outcomes, including en bloc resection, complete resection, and curative resection rates, and long-term outcomes, including recurrence and mortality rates, were extracted and analyzed. Therapeutic outcomes, divided by pre-ER indication and post-ER criteria, were evaluated to identify the suitability of current indications.

\section{Histologic definitions}

En bloc resection was defined as complete removal of cancer in a single piece without fragmentation. Complete resection was defined as removal of cancer with no neoplastic components at the lateral or vertical margins and without lymphovascular invasion on microscopic examination. Curative resection was defined as removal of cancer with $20 \mathrm{~mm}$ or smaller intramucosal lesions without ulceration, neoplastic components at the lateral or vertical margins, and lymphovascular invasion. ${ }^{3}$ Recurrence was defined as reappearance of cancer at the site of the lesion (local recurrence) or synchronous, metachronous, or distant metastatic lesions.

\section{Statistical analysis}

Continuous variables were expressed as the median and interquartile range (IQR) because they were not normally distributed. Categorical variables were expressed as numbers and percentages. Mann-Whitney and Fisher's exact tests were used to compare two variables. In this study, a $p$-value $<0.05$ (2-tailed) was adopted as the threshold of statistical significance for all tests. The analyses were performed using SPSS version 20.0 (SPSS Inc., Chicago, IL, USA).

\section{Ethics statement}

This study was conducted according to the principles expressed in the Declaration of Helsinki, and approved by the Institutional Review Board of Chuncheon Sacred Heart Hospital (2016-87).

\section{RESULTS}

\section{Clinical and endoscopic characteristics of the en- rolled population}

From 2006 to 2015, 275 EGCs with undifferentiated-type histology from 275 patients were identified. Of the total lesions, 186 were PDC (67.6\%) and 89 were SRC (32.4\%). The median age of the enrolled population was 69 years (IQR, $60-76$ years), with a male predominance (165 men, $60 \%$ ). The 
Table 1. Clinical and Endoscopic Characteristics of the Enrolled Population ( $n=275)$

\begin{tabular}{|c|c|}
\hline Age, yr, median (IQR) & $69(60-76)$ \\
\hline Sex, men: women $(\%)$ & 165 (60\%): $110(40 \%)$ \\
\hline \multicolumn{2}{|l|}{ Tumor location (\%) } \\
\hline Antrum & $131(47.6 \%)$ \\
\hline Low body & $83(30.2 \%)$ \\
\hline Mid body & $28(10.2 \%)$ \\
\hline Upper body, cardia & $33(12 \%)$ \\
\hline \multicolumn{2}{|l|}{ Macroscopic type (\%) } \\
\hline Elevated & $101(36.7 \%)$ \\
\hline Flat & $98(35.6 \%)$ \\
\hline Depressed & $76(27.6 \%)$ \\
\hline Lesion size, pre-ER measurement (mm), median (IQR) & $25(15-40)$ \\
\hline Lesion size, post-ER measurement (mm), median (IQR) & $18(11-26)$ \\
\hline \multicolumn{2}{|l|}{ Depth of invasion } \\
\hline Mucosal lesion & $176(64 \%)$ \\
\hline Submucosal lesion & $99(36 \%)$ \\
\hline \multicolumn{2}{|l|}{ Histology, pre-ER (\%) } \\
\hline Adenocarcinoma, poorly differentiated & $89(32.4 \%)$ \\
\hline Signet ring cell carcinoma & $59(21.5 \%)$ \\
\hline Adenocarcinoma, moderately differentiated & $55(20 \%)$ \\
\hline Adenocarcinoma, well differentiated & $32(11.6 \%)$ \\
\hline Low-grade dysplasia & $15(5.5 \%)$ \\
\hline High-grade dysplasia & $13(4.7 \%)$ \\
\hline Atypical cells or unknown & $12(4.4 \%)$ \\
\hline \multicolumn{2}{|l|}{ Histology, post-ER (\%) } \\
\hline Adenocarcinoma, poorly differentiated & $186(67.6 \%)$ \\
\hline Signet ring cell carcinoma & $89(32.4 \%)$ \\
\hline \multicolumn{2}{|l|}{ Resection method (\%) } \\
\hline ESD & $250(90.9 \%)$ \\
\hline EMR & $25(9.1 \%)$ \\
\hline \multicolumn{2}{|l|}{ Pre-ER indication } \\
\hline Expanded indication & $82(29.8 \%)$ \\
\hline Beyond expanded indication & $193(70.2 \%)$ \\
\hline \multicolumn{2}{|l|}{ Post-ER criteria } \\
\hline Expanded criteria & $108(39.3 \%)$ \\
\hline Beyond expanded criteria & $167(60.7 \%)$ \\
\hline \multicolumn{2}{|l|}{ Helicobacter pylori infection (\%) } \\
\hline Uninfected & $82(29.8 \%)$ \\
\hline Infected & $110(40 \%)$ \\
\hline Unknown & $83(30.2 \%)$ \\
\hline Follow-up duration, yr, median (IQR) & $3.96(1.11-5.09)$ \\
\hline Lost to follow-up (\%) & $17(6.2 \%)$ \\
\hline
\end{tabular}

IQR, interquartile range; ER, endoscopic resection; ESD, endoscopic submucosal dissection; EMR, endoscopic mucosal resection. 
Table 2. Immediate Therapeutic Outcomes according to Pre-ER Indication

\begin{tabular}{|c|c|c|c|c|}
\hline & $\begin{array}{l}\text { Overall outcomes } \\
\qquad(n=275)\end{array}$ & $\begin{array}{c}\text { Outcomes in lesions } \\
\text { with expanded } \\
\text { indication }(n=82)\end{array}$ & $\begin{array}{l}\text { Outcomes in lesions } \\
\text { with beyond expanded } \\
\text { indication }(n=193)\end{array}$ & $p$-value \\
\hline En bloc resection & $254(92.4 \%)$ & $77(93.9 \%)$ & $177(91.7 \%)$ & 0.63 \\
\hline Complete resection & $220(80 \%)$ & $68(82.9 \%)$ & $152(78.8 \%)$ & 0.51 \\
\hline Curative resection & $100(36.4 \%)$ & $40(48.8 \%)$ & $60(31.1 \%)$ & 0.006 \\
\hline Resection margin involvement & $42(15.3 \%)$ & $10(12.2 \%)$ & $32(16.6 \%)$ & 0.41 \\
\hline Lateral margin & $14(5.1 \%)$ & $4(4.9 \%)$ & $10(5.2 \%)$ & \\
\hline Vertical margin & $21(7.6 \%)$ & $6(7.3 \%)$ & $15(7.8 \%)$ & \\
\hline Both margin & $7(2.5 \%)$ & $0(0 \%)$ & $7(3.6 \%)$ & \\
\hline Depth of invasion & & & & 0.78 \\
\hline Mucosal lesion & $176(64 \%)$ & $54(65.9 \%)$ & $122(63.2 \%)$ & \\
\hline Submucosal lesion & $99(36 \%)$ & $28(34.1 \%)$ & $71(36.8 \%)$ & \\
\hline Lymphovascular invasion & $26(9.5 \%)$ & $6(7.3 \%)$ & $20(10.4 \%)$ & 0.51 \\
\hline \multicolumn{5}{|l|}{ Complications } \\
\hline Delayed bleeding ${ }^{a}$ & $2(0.7 \%)$ & $0(0 \%)$ & $2(1.0 \%)$ & 0.12 \\
\hline Perforation & $5(1.8 \%)$ & $3(3.7 \%)$ & $2(1.0 \%)$ & 0.25 \\
\hline Procedure time, min, median (IQR) & $50(33-76)$ & $45(31.8-64.3)$ & $60(33.5-88)$ & 0.02 \\
\hline
\end{tabular}

ER, endoscopic resection; IQR, interquartile range; ESD, endoscopic submucosal dissection.

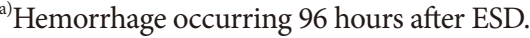

antrum was the most frequent location of lesions (47.6\%), followed by the lower body (30.2\%), upper body and cardia (12\%), and midbody (10.2\%). The macroscopic types of the lesions were elevated (36.7\%), flat (35.6\%), and depressed (27.6\%). The median pre-ER endoscopic size measurement of the lesions was $25 \mathrm{~mm}$ (IQR, 15-40 mm) and the median post-ER histologic size measurement was $18 \mathrm{~mm}$ (IQR, 11-26 mm). With regard to the depth of invasion, $64 \%$ were mucosal lesions and $36 \%$ exhibited submucosal invasion. The pre-ER histology was variable and included PDC (32.4\%), SRC (21.5\%), moderately differentiated adenocarcinoma (20\%), well-differentiated adenocarcinoma (11.6\%), low-grade dysplasia (5.5\%), high-grade dysplasia (4.7\%), and atypical cells or unknown (4.4\%). The vast majority of lesions were resected using the ESD method (90.9\%), and $9.1 \%$ of lesions were resected using the EMR method. Helicobacter pylori were detected in $40 \%$ of the enrolled population.

In terms of the pre-ER indications, only 82 lesions (29.8\%) were included in the expanded indication group, and 193 lesions $(70.2 \%)$ were included in the beyond expanded indication group. In the post-ER histologic criteria, 108 lesions (39.3\%) and 167 lesions (60.7\%) fulfilled the expanded and beyond expanded criteria, respectively. There was discrepancy between pre-ER indications and post-ER criteria in 36.7\% (101/275) of all lesions. The clinical and endoscopic characteristics of the enrolled population are summarized in Table 1 .

\section{Immediate therapeutic outcomes according to pre- ER indications and post-ER criteria}

The immediate therapeutic outcomes were as follows: en bloc resection rate: $92.4 \%$; complete resection rate: $80 \%$; and curative resection rate: $36.4 \%$. Lesions with pre-ER expanded indication were associated with better therapeutic outcomes than those of lesions with pre-ER beyond expanded indication, although significant differences between these two groups were detected only with regard to the curative resection rate $(48.8 \%$ vs. $31.1 \%, p=0.006)$. The submucosal invasion rate was high regardless of indications, which was $34.1 \%$ in lesions with pre-ER expanded indication and 36.8\% in lesions with pre-ER beyond expanded indication. The lymphovascular invasion rates were $7.3 \%$ and $10.4 \%$ in lesions with pre-ER expanded indication and lesions with pre-ER beyond expanded indication, respectively $(p=0.51)$. The procedure-related complication rates were $0.7 \%$ for delayed bleeding and $1.8 \%$ for gastric perforation. These rates were not significantly different between lesions with pre-ER expanded indication and beyond expanded indication. The median procedure time was 50 minutes (IQR, 33-76 minutes), and lesions with pre-ER beyond expanded indication exhibited longer procedure time than that exhibited by lesions with pre-ER expanded indication (60 minutes vs. 45 minutes, $p=0.02$ ). Detailed immediate therapeutic outcomes according to the pre-ER indication are described in Table 2. 
Table 3. Immediate Therapeutic Outcomes according to the Post-ER Criteria

\begin{tabular}{|c|c|c|c|c|}
\hline & $\begin{array}{l}\text { Overall outcomes } \\
\qquad(n=275)\end{array}$ & $\begin{array}{l}\text { Outcomes in lesions } \\
\text { with expanded criteria } \\
\qquad(n=109)\end{array}$ & $\begin{array}{c}\text { Outcomes in lesions } \\
\text { with beyond expanded } \\
\text { criteria }(n=166)\end{array}$ & $p$-value \\
\hline En bloc resection & $254(92.4 \%)$ & $104(95.4 \%)$ & $150(90.4 \%)$ & 0.16 \\
\hline Complete resection & $220(80 \%)$ & $100(91.7 \%)$ & $120(72.3 \%)$ & $<0.001$ \\
\hline Curative resection & $100(36.4 \%)$ & $100(91.7 \%)$ & Not applicable & Not applicable \\
\hline Resection margin involvement & $42(15.3 \%)$ & $5(4.6 \%)$ & $37(22.3 \%)$ & $<0.001$ \\
\hline Lateral margin & $14(5.1 \%)$ & $3(2.8 \%)$ & $11(6.6 \%)$ & \\
\hline Vertical margin & $21(7.6 \%)$ & $2(1.8 \%)$ & $19(11.4 \%)$ & \\
\hline Both margin & $7(2.5 \%)$ & $0(0 \%)$ & $7(4.2 \%)$ & \\
\hline Depth of invasion & & & & $<0.001$ \\
\hline Mucosal lesion & $176(64 \%)$ & $109(100 \%)$ & $67(40.4 \%)$ & \\
\hline Submucosal lesion & $99(36 \%)$ & $0(0 \%)$ & $99(59.6 \%)$ & \\
\hline Lymphovascular invasion & $26(9.5 \%)$ & $4(3.7 \%)$ & $22(13.3 \%)$ & 0.01 \\
\hline \multicolumn{5}{|l|}{ Complications } \\
\hline Delayed bleeding ${ }^{\text {a) }}$ & $2(0.7 \%)$ & $0(0 \%)$ & $2(1.2 \%)$ & 0.49 \\
\hline Perforation & $5(1.8 \%)$ & $1(0.9 \%)$ & $4(2.4 \%)$ & 0.17 \\
\hline Procedure time, min, median (IQR) & $50(33-76)$ & $41(29-70)$ & $59(37.8-80)$ & 0.002 \\
\hline
\end{tabular}

ER, endoscopic resection; IQR, interquartile range; ESD, endoscopic submucosal dissection.

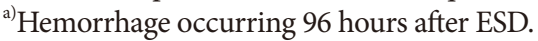

Compared to lesions with post-ER beyond expanded criteria, those with post-ER expanded criteria were associated with better therapeutic outcomes. The en bloc resection rate was not significantly different between these two groups (95.4\% vs. $90.4 \%, p=0.16$ ). However, complete resection rate was higher in the lesions with expanded criteria than in lesions with beyond expanded criteria ( $91.7 \%$ vs. $72.3 \%, p<0.001)$. Of the lesions with post-ER beyond expanded criteria, 59.6\% exhibited submucosal invasion. The lymphovascular invasion rate was $3.7 \%$ in lesions with post-ER expanded criteria and 13.3\% in lesions with post-ER beyond expanded criteria $(p=0.01)$. Procedure-related complication rates were not significantly different between lesions with post-ER expanded criteria and beyond expanded criteria. Lesions with post-ER beyond expanded criteria had a longer procedure time than that of lesions with post-ER expanded criteria (59 minutes vs. 41 minutes, $p=0.002$ ). Detailed immediate therapeutic outcomes according to the post-ER criteria are described in Table 3.

\section{Immediate therapeutic outcomes according to the histology}

There was no difference in immediate outcomes between PDC and SRC, including en bloc resection, complete resection, and curative resection rate. However, ER of PDC was associated with vertical margin involvement $(9.7 \%$ vs. $3.4 \%)$ while ER of SRC was associated with lateral margin involvement
(6.7\% vs. $4.3 \%)$, although these associations were not statistically significant $(p=0.21)$. ER of PDC rather than SRC was more strongly associated with submucosal invasion $(41.9 \%$ vs. $23.6 \%, p=0.003$ ). The rate of lymphovascular invasion was also higher in ER of PDC than of SRC, although this difference was statistically insignificant ( $11.3 \%$ vs. $5.6 \%, p=0.19)$. The rate of procedure-related complications including delayed bleeding and gastric perforation was not significantly different between ER of PDC and SRC ( $p=0.54 \& p=0.70$, respectively). The procedure time was not significantly different between ER of PDC and SRC (50 minutes vs. 50 minutes, $p=0.94$ ). Detailed immediate therapeutic outcomes according to the histology are described in Table 4.

\section{Long-term therapeutic outcomes}

Fig. 1 shows the treatment flow of ER of EGC with undifferentiated-type histology. With regard to the long-term outcomes, the recurrence rate was $10.2 \%$ and median recurrence period was 13.6 months (IQR, 2.4-47.7 months) after ER. A total of $5(1.8 \%)$ deaths were observed in the overall population during the observational period (median follow up: 3.96 years), and these were not related to cancer (Table 5). All of the recurrence cases were treated by repeated ESD or operation, and there was no recurrence at the last follow-up (Fig. 1).

In terms of the recurrence rate, there was no difference between lesions with curative resection and non-curative resec- 
Table 4. Immediate Therapeutic Outcomes according to the Histology

\begin{tabular}{|c|c|c|c|c|}
\hline & $\begin{array}{l}\text { Overall outcomes } \\
\qquad(n=275)\end{array}$ & $\begin{array}{c}\text { Outcomes in poorly } \\
\text { differentiated } \\
\text { adenocarcinoma }(n=186)\end{array}$ & $\begin{array}{l}\text { Outcomes in signet ring } \\
\text { cell carcinoma }(n=89)\end{array}$ & $p$-value \\
\hline En bloc resection & $254(92.4 \%)$ & $171(91.9 \%)$ & $83(93.3 \%)$ & 0.81 \\
\hline Complete resection & $220(80 \%)$ & $146(78.5 \%)$ & $74(83.1 \%)$ & 0.42 \\
\hline Curative resection & $100(36.4 \%)$ & $67(36 \%)$ & $33(37.1 \%)$ & 0.89 \\
\hline Resection margin involvement & $42(15.3 \%)$ & $30(16.1 \%)$ & $12(13.5 \%)$ & 0.21 \\
\hline Lateral margin & $14(5.1 \%)$ & $8(4.3 \%)$ & $6(6.7 \%)$ & \\
\hline Vertical margin & $21(7.6 \%)$ & $18(9.7 \%)$ & $3(3.4 \%)$ & \\
\hline Both margin & $7(2.5 \%)$ & $4(2.2 \%)$ & $3(3.4 \%)$ & \\
\hline Depth of invasion & & & & 0.003 \\
\hline Mucosal lesion & $176(64 \%)$ & $108(58.1 \%)$ & $68(76.4 \%)$ & \\
\hline Submucosal lesion & $99(36 \%)$ & $78(41.9 \%)$ & $21(23.6 \%)$ & \\
\hline Lymphovascular invasion & $26(9.5 \%)$ & $21(11.3 \%)$ & $5(5.6 \%)$ & 0.19 \\
\hline \multicolumn{5}{|l|}{ Complications } \\
\hline Delayed bleeding ${ }^{\mathrm{a})}$ & $2(0.7 \%)$ & $1(0.5 \%)$ & $1(1.1 \%)$ & 0.54 \\
\hline Perforation & $5(1.8 \%)$ & $3(1.6 \%)$ & $2(2.2 \%)$ & 0.70 \\
\hline Procedure time, min, median (IQR) & $50(33-76)$ & $50(33-72.3)$ & $50(30-83.5)$ & 0.94 \\
\hline
\end{tabular}

IQR, interquartile range; ESD, endoscopic submucosal dissection.

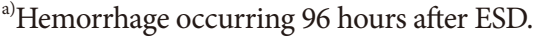

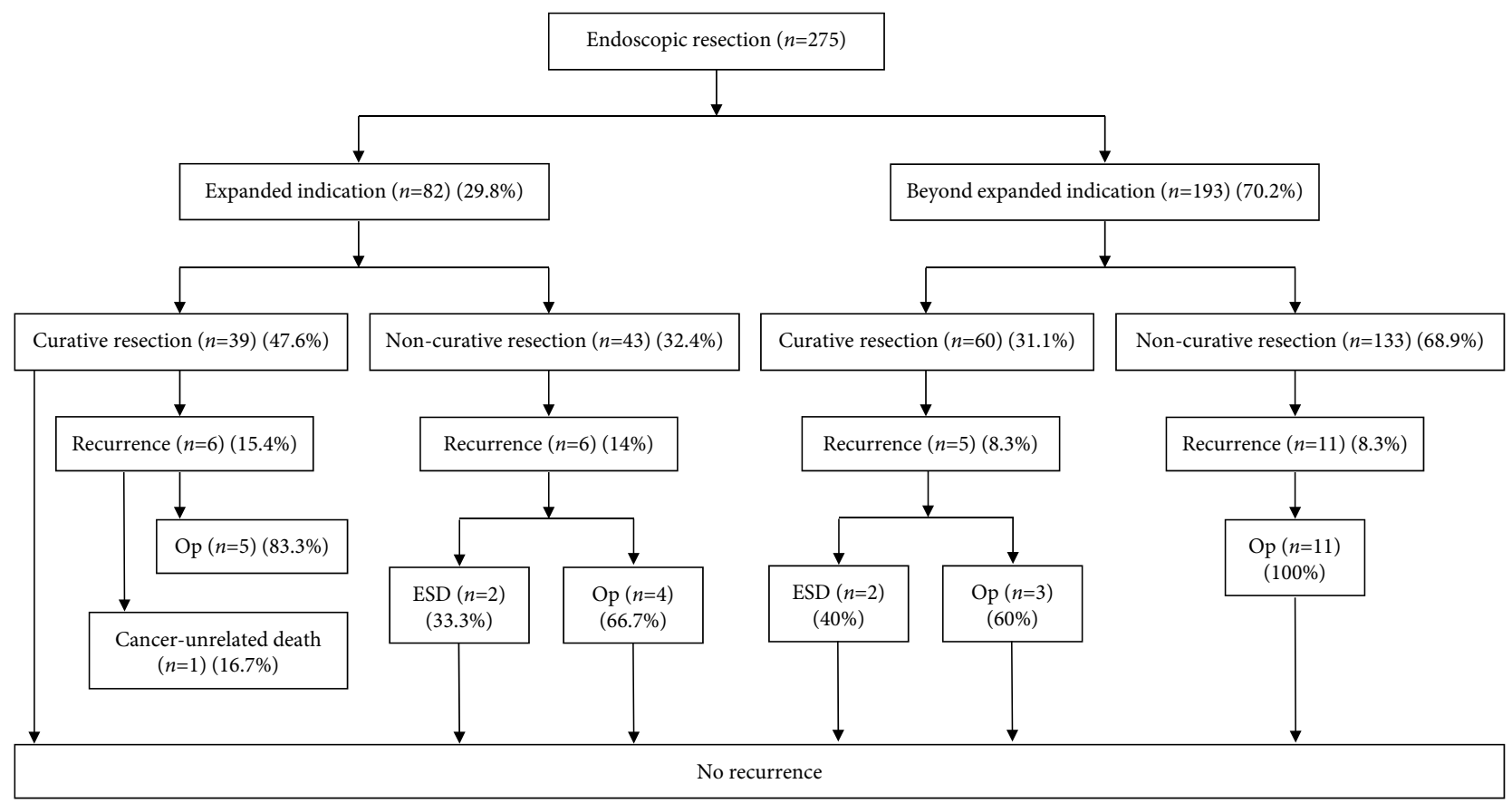

Fig. 1. Treatment flow of endoscopic resection of early gastric cancer with undifferentiated-type histology. ESD, endoscopic submucosal dissection; Op, operation.

tion ( $p=0.28)$. This rate was also not different between PDC and SRC ( $p=0.11)$. Although lesions with pre-ER expanded indication showed higher recurrence rates than those with pre-
ER beyond expanded indication ( $14.6 \%$ vs. $8.3 \%, p=0.02)$, this rate was not statistically significant $(9.2 \%$ vs. $10.8 \%, p=0.21)$ (Table 5). 


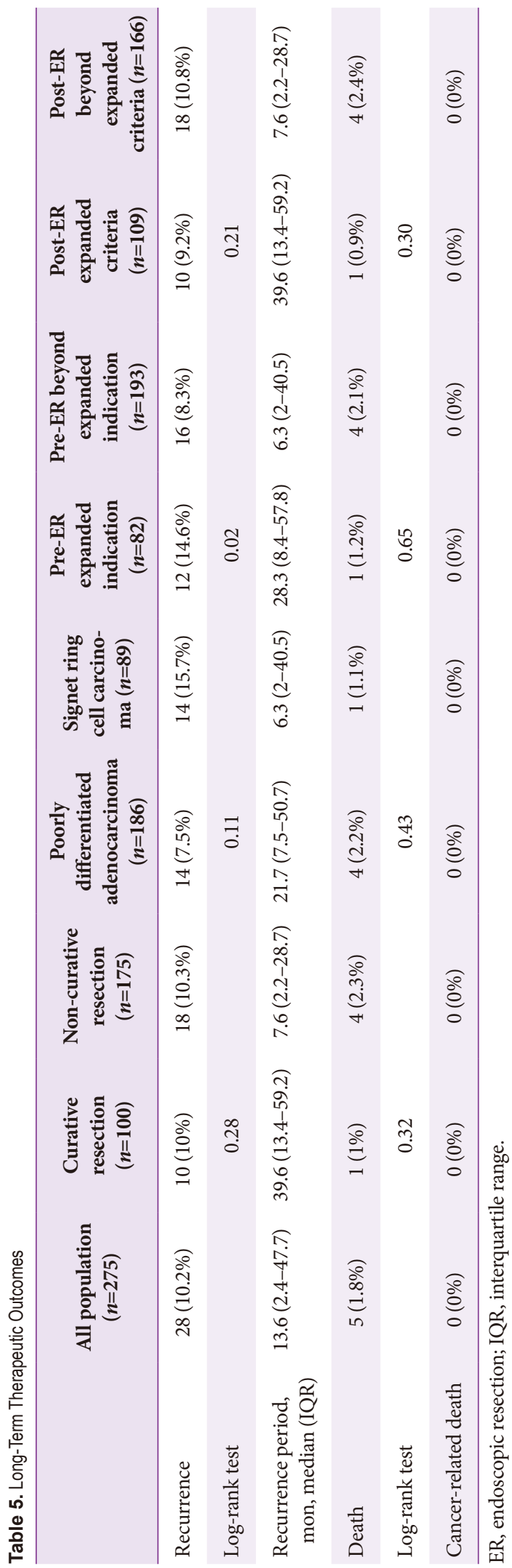

In terms of the mortality rate, there was no difference between lesions with curative resection and non-curative resection $(p=0.32)$. This rate was not different between PDC and SRC ( $p=0.43)$. Mortality rates according to the pre-ER indication and post-ER criteria showed no significant differences ( $p=0.65$ and $p=0.30$, respectively) (Table 5).

\section{DISCUSSION}

Previous studies evaluating the therapeutic outcomes of ER of EGC with undifferentiated-type histology have focused on post-ER histology (divided by PDC and SRC) or have included lesions that met only the expanded indication or criteria, which could have exaggerated the therapeutic outcomes. ${ }^{9-11}$ However, the proportion of cases with histologic discrepancy (EGCs with undifferentiated-type histology that were initially diagnosed as differentiated-type EGC, dysplasia, or atypical glands) is known to be high $(84.7 \%),{ }^{12}$ and the size discrepancy between pretreatment endoscopic measurements and those of the resected specimens is known to be higher in EGC with undifferentiated-type histology than in differentiated-type EGC. ${ }^{13}$ Owing to the unpredictability of the depth of invasion and lateral margin of EGC with undifferentiated-type histology, we included all cases of resected EGC with undifferentiated-type histology regardless of the indication or criteria. In this study, histologic discrepancies were observed in 54.9\% (151/275) of the lesions, and between pre-ER indication and post-ER criteria, in 36.7\% $(101 / 275)$ of the lesions. Therefore, most lesions of EGC (70.2\%) were resected under the beyond-expanded indication, and only $39.3 \%$ of lesions fulfilled the expanded criteria, leading to a low curative resection rate (Table 1).

However, irrespective of the pre-ER indication, ER of EGCs with undifferentiated-type histology showed acceptable en bloc, complete resection, and procedure-related complication rates, implicating a technically feasible treatment option (Table 2). Despite the technical feasibility of $\mathrm{ER}$, endoscopists could not accurately predict the resection margin, depth of invasion, and lymphovascular invasion in lesions with expanded indication. Of the lesions with preER expanded indication, $12.2 \%$ showed involvement of the resection margin and $34.1 \%$ exhibited submucosal invasion in the final pathology, which is a relatively high value even after considering the inaccuracy of prediction through visual inspection. For accurate prediction of ambiguous margins and depth of invasion in EGC with undifferentiated-type histology, magnifying endoscopy, narrow-band imaging, and confocal endomicroscopy have frequently been suggested as supplementary methods. ${ }^{14}$ Although the results of stud- 
ies using these methods are encouraging, current evidence supporting the applications of these modalities before ER are limited.

Lesions with pre-ER expanded indication were associated with better therapeutic outcomes than those of lesions with pre-ER beyond expanded indication, although significant difference was confirmed only in the curative resection rate $(48.8 \%$ vs. $31.1 \%, p=0.006)$. Among lesions with pre-ER beyond expanded indication, $16.6 \%$ exhibited involvement of the resection margin and $36.8 \%$ exhibited submucosal invasion. The lymphovascular invasion rate was also high at $10.4 \%$, although statistical significance was not found (vs. lesions with pre-ER expanded indication) (Table 2). Therefore, at least when PDC or SRC is confirmed in pre-ER biopsy, ER confined to expanded indication lesions can be considered for the treatment of EGC with undifferentiated-type histology, owing to the uncertainties in ambiguous margins and risk of lymphovascular invasion in EGC with undifferentiated-type histology.

The proposed expanded criteria for ER of EGC with undifferentiated-type histology were based on a retrospective review of large surgical data, and focused on the risk of lymph node metastasis. ${ }^{3,45}$ Our study also found favorable outcomes in lesions fulfilling post-ER expanded criteria. The curative resection rate was $91.7 \%$, and only $4.6 \%$ of the lesions showed involvement of the resection margin. The rate of lymphovascular invasion was $3.7 \%$ (vs. 13.3\%), which is a significantly lower value compared to that observed in lesions with beyond expanded criteria (Table 3).

In terms of the histology, ER of PDC was associated with vertical margin involvement, and ER of SRC was associated with lateral margin involvement, which is consistent with the results of a previous study. ${ }^{9}$ The rate of lymphovascular invasion was also higher in ER of PDC than SRC (Table 4). Considering the different origins and spreading patterns of PDC and SRC, separate indications might be required, although current guidelines recommend different treatment strategies only for EGC with differentiated- and undifferentiated-type histology. ${ }^{4,5,16}$

With regard to long-term outcomes, there were no statistically significant differences between lesions with curative and non-curative resection in terms of the recurrence or mortality rates. There were also no significant differences between PDC and SRC or between lesions with post-ER expanded criteria and beyond expanded criteria. These results are also consistent with those of previous studies. ${ }^{9,10}$ However, the recurrence period was relatively short in lesions with non-curative resection, SRC, and beyond expanded criteria in our study (Table 5). This could be attributed to local recurrence owing to overlooked or missed lesions around the main EGC or to incorrect prediction of the depth or lateral margin of EGC with undifferentiated-type histology. It may be possible to find statistically significant differences in studies with larger populations or longer durations. All recurrent cases were treated by repeated ESD or operation, and no recurrence was observed at the last follow-up (Fig. 1).

To the best of our knowledge, this is the first multicenter study using a database to examine EGC with undifferentiated-type histology in Korea. We identified pre-ER indications in all of the cases, and compared the therapeutic outcomes between lesions with pre-ER indications and post-ER criteria. Despite these strengths, selection bias could be a limitation owing to the retrospective nature of this study. Another limitation was that the endoscopic images of all patients were not accessible. This was particularly limiting in cases of lesions treated by non-curative resection, or recurrence, which made it difficult to confirm whether ER could be considered.

In conclusion, ER, which is confined to expanded indication lesions, can be considered for the treatment of EGC with undifferentiated-type histology.

Conflicts of Interest

The authors have no financial conflicts of interest.

\section{Author contributions}

Conceptualization: Chang Seok Bang, Jae Myung Park, Gwang Ho Baik, Jong Jae Park

Data curation: CSB, JMP, GHB, JJP, Moon Kyung Joo, Jae Young Jang, Seong Woo Jeon, Suck Chei Choi, Jae Kyu Sung, Kwang Bum Cho

Formal analysis: CSB, GHB

Supervision: GHB, JJP

Writing-original draft: CSB

Writing-review\&editing: GHB, JJP

\section{REFERENCES}

1. Gotoda T. Endoscopic resection of early gastric cancer. Gastric Cancer 2007;10:1-11.

2. Soetikno R, Kaltenbach T, Yeh R, Gotoda T. Endoscopic mucosal resection for early cancers of the upper gastrointestinal tract. J Clin Oncol 2005;23:4490-4498.

3. Gotoda T, Yanagisawa A, Sasako M, et al. Incidence of lymph node metastasis from early gastric cancer: estimation with a large number of cases at two large centers. Gastric Cancer 2000;3:219-225.

4. Japanese Gastric Cancer Association. Japanese gastric cancer treatment guidelines 2010 (ver. 3). Gastric Cancer 2011;14:113-123.

5. Lee JH, Kim JG, Jung HK, et al. Clinical practice guidelines for gastric cancer in Korea: an evidence-based approach. J Gastric Cancer 2014;14:87-104.

6. Katsube T, Konnno S, Hamaguchi K, et al. The efficacy of endoscopic mucosal resection in the diagnosis and treatment of group III gastric lesions. Anticancer Res 2005;25:3513-3516.

7. Bang CS, Baik GH, Shin IS, et al. Endoscopic submucosal dissection for early gastric cancer with undifferentiated-type histology: a meta-analysis. World J Gastroenterol 2015;21:6032-6043.

8. Ajani JA, D’Amico TA, Almhanna K, et al. Gastric cancer, version 3.2016, 
NCCN clinical practice guidelines in oncology. J Natl Compr Canc Netw 2016;14:1286-1312.

9. Kim JH, Kim YH, Jung da $\mathrm{H}$, et al. Follow-up outcomes of endoscopic resection for early gastric cancer with undifferentiated histology. Surg Endosc 2014;28:2627-2633.

10. Ahn JY, Park HJ, Park YS, et al. Endoscopic resection for undifferentiated-type early gastric cancer: immediate endoscopic outcomes and longterm survivals. Dig Dis Sci 2016;61:1158-1164.

11. Oka S, Tanaka S, Higashiyama M, et al. Clinical validity of the expanded criteria for endoscopic resection of undifferentiated-type early gastric cancer based on long-term outcomes. Surg Endosc 2014;28:639-647.

12. Min BH, Kang KJ, Lee JH, et al. Endoscopic resection for undifferentiated early gastric cancer: focusing on histologic discrepancies between forceps biopsy-based and endoscopic resection specimen-based diagno- sis. Dig Dis Sci 2014;59:2536-2543.

13. Kang HY, Kim SG, Kim JS, Jung HC, Song IS. Clinical outcomes of endoscopic submucosal dissection for undifferentiated early gastric cancer. Surg Endosc 2010;24:509-516.

14. Gong S, Xue HB, Ge ZZ, et al. Value of magnifying endoscopy with narrow-band imaging and confocal laser endomicroscopy in detecting gastric cancerous lesions. Medicine (Baltimore) 2015;94:e1930.

15. Hirasawa T, Gotoda T, Miyata S, et al. Incidence of lymph node metastasis and the feasibility of endoscopic resection for undifferentiated-type early gastric cancer. Gastric Cancer 2009;12:148-152.

16. Kumarasinghe MP, Lim TK, Ooi CJ, Luman W, Tan SY, Koh M. Tubule neck dysplasia: precursor lesion of signet ring cell carcinoma and the immunohistochemical profile. Pathology 2006;38:468-471 